\title{
Coincidence of Guillain-Barré syndrome presenting with Landry's acute flaccid paralysis and transverse myelitis
}

\author{
Desislava Kalinova, Rasho Rashkov \\ Clinic of Rheumatology, UMHAT St. Ivan Rilski University Hospital, Sofia, Bulgaria
}

\begin{abstract}
Transverse myelitis is one of the causes of acute transverse myelopathy; three main categories are described in the differential diagnosis of transverse myelitis: demyelination (multiple sclerosis, neuromyelitis optica), infections (herpes zoster and herpes simplex virus), and some autoimmune connective tissue disorders (systemic lupus erythematosus, vasculitis). The authors present a clinical case of a 33-year-old patient with transverse myelitis occurring in the course of acute inflammatory demyelinating polyradiculoneuropathy (Guillain-Barré syndrome). The patient's medical history was notable. The patient was diagnosed with thrombotic thrombocytopenic purpura (Moschcowitz syndrome) and leukocytoclastic vasculitis when he was 12 years old.
\end{abstract}

Key words: Guillain-Barré syndrome, transverse myelitis, leukocytoclastic vasculitis, Moschcowitz syndrome, polyradiculoneuropathy.

\section{Introduction}

Transverse myelitis is one of the causes of acute transverse myelopathy; as three main categories: 1) demyelination (multiple sclerosis, neuromyelitis optica), 2) infections (herpes zoster and herpes simplex virus), 3) autoimmune connective tissue diseases (systemic lupus erythematosus, vasculitis). We present a clinical case of a 33-year-old patient with coincidence of Guillain-Barré syndrome presenting with Landry's acute flaccid paralysis and transverse myelitis. The described patient was diagnosed with thrombotic thrombocytopenic purpura (Moschcowitz syndrome) and leukocytoclastic vasculitis in childhood.

\section{Case report}

A 33-year-old man was admitted to the emergency department in the summer of 2014 with a fever (up to $38-38.5^{\circ} \mathrm{C}$ ), dysuria, and urine retention. A urethral catheter was inserted, with resolution of the retention, and the patient was referred to a urological unit. The diagnosis of prostatitis was established, and the patient started antibiotic treatment. Three days later, the patient reported unstable gait, numbness, and weakness in the distal parts of the upper and lower limbs. The weakness in the extremities had an ascending form, progressive course, up to quadriparesis in the subsequent 24 hours. The patient was admitted to the department of neurology because of the neurological nature of the symptoms.

On admission the neurological examination showed the following:

- moderate to severe central paraparesis of the lower limbs and latent central paresis of the upper limbs,

- $\mathrm{Th}_{3}-\mathrm{Th}_{4}$ sensory level,

- pelvic reservoir dysfunction manifested as urine retention.

Three days later additional neurological symptoms appeared: bilateral peripheral lesions of facial nerve, more pronounced in the left side, lesion of the left abducens nerve, transient dysphagia, and dysarthria.

Laboratory tests revealed increased inflammatory markers such as erythrocyte sedimentation rate (ESR), C-reactive protein (CRP), fibrinogen and thrombocytopaenia $\left(90-114 \times 10^{9} /\right.$ l), and borderline serum creatinine (138-147 $\mu \mathrm{mol} / \mathrm{l})$. A lumbar puncture was performed and the cerebrospinal fluid (CSF) was examined. It showed increased total protein $1.55 \mathrm{~g} / \mathrm{l}$ (reference level $0.15-$ $0.45 \mathrm{~g} / \mathrm{l})$, normal cell count, and normal glucose level.

Address for correspondence:

Desislava Kalinova, Clinic of Rheumatology, UMHAT St. Ivan Rilski University Hospital, 13 Urvich St., 1612 Sofia, Bulgaria,

e-mail: d_kalinova666@abv.bg

Submitted: 2.01.2019; Accepted: 25.04.2019 
The CSF electrophoresis was notable for high $\gamma$-fraction without mono- or oligoclonal bands. Microbiological studies were negative for enterovirus, West Nile virus, tick-borne encephalitis, human immunodeficiency virus 1, 2 (HIV-1; HIV-2), Herpes simplex virus 1 and 2, Varicella zoster virus, and cytomegalovirus. Nasal, throat, urine, and hemocultures were also negative.

For examinations of the brain, face, eyes, ears, spine, and spinal cord a magnetic-resonance tomography (MRT) was performed, which showed altered signal along the $T_{1}-T_{4}$ segment. At the same level, edema in the white and grey matter of the myelin was noted. After contrast application, a disruption of the blood-brain barrier was observed at leve $\mathrm{C}_{5}-\mathrm{T}_{4}$. Brain MRT assessment revealed altered signal in the thalamus and left middle cerebellar peduncle - changes associated with increased fluid content. There was no bloodbrain barrier disruption after contrast administration.

The changes in the spinal cord and the brain were interpreted as inflammatory demyelinating condition acute disseminated encephalomyelitis/transversal myelitis in the active phase.

The electroneuromyography (ENMG) demonstrated polyneuropathy - demyelinating type with secondary axonal damage of the sensory and motor nerves. These findings were consistent with acute inflammatory polyneuropathy.

The differential diagnosis included the following:

- acute inflammatory demyelinating polyradiculoneuropathy, Guillain-Barré syndrome, presenting with Landry's acute flaccid (ascending) paralysis and transverse myelitis,

- Miller-Fisher syndrome, characterized by external ophthalmoplegia, ataxia, and areflexia,

- acute disseminated encephalitis and transversal myelitis during the course of a viral infection.

It was decided to treat with glucocorticosteroids (GC); methylprednisolone was administrated at a dose of $1 \mathrm{mg} / \mathrm{kg}$ intravenous application, and in the next three days pulse therapy was carried out with methylprednisolone $1000 \mathrm{mg}$ i.v.; immunoglobulins (800 mg/kg i.v.), symptomatic treatment - galantamine with vitamins from group $B$ as benfotiamine $\left(B_{1}\right)$, pyridoxine $\left(B_{6}\right)$, and cyanocobalamin $\left(B_{12}\right)$ supplementation, antibiotics, unfractionated heparin, and antihypertensive medications were also used. The neurological abnormalities ameliorated as a result of the applied treatment - resolution of the cranial nerve lesions concentrated the sensory disturbances in the distal parts of the limbs and improvement of the muscle strength in the upper limbs.

The patient's medical history was notable for an adverse reaction to hepatitis $B$ vaccination when he was 12 years old. At that time, he developed fever, urticaria on the trunk and the limbs, palpable purpura on the lower legs, myalgias, and hepatosplenomegaly. This episode was followed by acute renal failure, widespread suffusions, and generalized grand-mal type seizures. The skin biopsy of the lower leg revealed leukocytoclastic vasculitis (perivascular and mural infiltrates consisting of mononuclear and neutrophil leukocytes and erythrocyte extravasates involving the superficial venules and arterioles). Immunofluorescent staining demonstrated deposition of $\lg M, \lg A, C_{4}$, and fibrinogen in the vessel wall. A kidney biopsy was also performed with a histological picture of thrombotic microangiopathy. The patient was diagnosed with thrombotic thrombocytopenic purpura (TTP syndrome, Moschcowitz syndrome) which is a rare blood disorder characterized by clotting in small blood vessels of the body (thromboses). In its full-blown form, the disease consists of the pentad of [1, 2]:

- microangiopathic hemolytic anemia,

- thrombocytopenic purpura,

- neurological abnormalities include hemiplegia, paresthesia, visual disturbances, aphasia, and seizures,

- fever,

- renal disease, presenting with acute renal failure.

In the congenital form of TTP, mutations in the gene encoding the protease ADAMTS13 have been described [2]. ADAMTS13 is a protease that is responsible for the breakdown of the ultra-large von Willebrand factor (vWF) multimers. In the more common sporadic form, antibodies against ADAMTS13 can be isolated in most patients [2]. The increase in circulating multimers of VWF increase platelet adhesion to areas of endothelial injury, particularly at arteriole-capillary junctions [2, 3].

The patient was treated with GC, plasmapheresis, and hemodialysis for 18 months. Kidney function improved, and the dose of the GC was tapered to discontinuation. Reduction in GC dose was beneficial due to the development of metabolic syndrome with hypertension, obesity, hyperuricemia, impaired glucose tolerance in this patient adolescent age.

One month after onset of the latest symptoms the patient was admitted to the clinic of rheumatology. A physical examination showed systolic murmur at the heart apex, propagating to the posterior axillar line, diastolic murmur with punctum maximum at the aortic valve, propagating to the carotid arteries, and bilateral knee arthritis. His neurological status revealed quadriparesis to paraplegia of the lower limbs - peripheral type, knee and Achilles areflexia, tactile hypesthesia in the upper limbs - distal type. The routine lab studies showed high ESR $(45 \mathrm{~mm})$, C-reactive protein $(40 \mathrm{~g} / \mathrm{l})$, leukocytosis with neutrophilia on background corticosteroid therapy, high BUN (15 g/l), and low-grade proteinuria up to $0.6 \mathrm{~g} / \mathrm{l}$ with normal concentration of creatinine $(67 \mathrm{mmol} / \mathrm{l})$.

Due to the occurrence of vasculitis in childhood, it has also been taken into account in differential diagno- 
sis polyarteritis nodosa (PAN) and systemic lupus erythematosus with involvement of the CNS presenting as transversal myelitis and disseminated encephalitis.

Polyarteritis nodosa is systemic necrotizing vasculitis predominantly affecting the middle-sized muscular arteries [4]. Given this definition, we performed a CT angiography of the abdomen and pelvis with no signs of stenoses, thromboses, or aneurysms of the abdominal aorta and its branches. Albeit rarely, PAN could affect the cerebral arteries with resulting ischemia, hemorrhage, and epileptic seizures [4]. A contrast-enhanced MRI of the brain was also performed with no evidence of aneurysms, stenoses, or thromboses of the blood vessels. The only finding was a gliotic focus of vascular origin, located supratentorially in the subcortical brain tissue of the left frontal area. An electrocardiography (ECG) showed Q-wave in leads II, III, and avF - a finding that had been persisting since childhood. We assumed that the patient may have had coronaritis due to Moschcowitz syndrome, so we performed an echocardiography that revealed pericardial effusion, apical septal hypokinesia, anterior mitral leaflet prolapse with resulting mitral regurgitation - second grade, and aortic regurgitation - first grade. A CT coronarography did not reveal any significant stenoses. A muscular band of the left anterior descending artery was noted. The above studies allowed for the exclusion of the PAN.

It is possible that transversal myelitis is a neurological manifestation of systemic lupus erythematosus [5-8]. This hypothesis is supported by the presence of arthritis, thrombocytopenia, low-grade proteinuria, and pericardial effusion. ANA testing by IFA (Indirect Immunofluorescence Assay) and immunoblotting for assessment of anti-RNP/Sm, Sm, Ro, La, Ro-52, Scl-70, PM/Scl, anti-Jo-1, anti-Cent. B, anti-PCNA, anti-nucleosomes, anti-histones, anti-Rib-P were all negative. The complement components C3: $1.85 \mathrm{~g} / \mathrm{l}$; C4: $0.35 \mathrm{~g} / \mathrm{l}$ were normal. The serum level of immunoglobulins $A, G$, and $M$ were also normal. The absence of specific SLE antibodies and anti-platelet antibodies and the uncertainty about the origin of the kidney damage made us exclude this diagnosis. The kidney changes may be a result of the TTP and could explain the low-grade proteinuria and the ultrasound findings of a diffuse parenchymal process.

The patient was tested for the presence of the following antibodies: anti-proteinase 3, anti-myeloperoxidase,

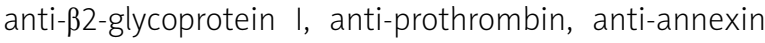
V-lgG, anti-annexin $\mathrm{V} \operatorname{IgM}$, anticardiolipin antibodies, and rheumatoid factor - IgM, IgA, IgG; all were negative. There were no Guillain-Barré syndrome specific antibodies (anti-GM1, GM2, GM3, GM4, GD1b, GD2, GD3, GT1a, GT1b, Gq1b).
Repeated CFS assessement revealed slightly increased protein - $0.65 \mathrm{~g} / \mathrm{l}(0.15-0.45 \mathrm{~g} / \mathrm{l})$, normocytosis.

Based on the clinical picture and results ENMG and MRI it determined that the patient had acute demyelinating polyradiculopathy, Guillain-Barré syndrome presenting with ascending paralysis type Landry and transversal myelitis. The GC therapy was continued with gradual tapering to maintenance dose of $8 \mathrm{mg}$ of methylprednisolone per day. It was also decided to continue administration of galantamine, benfotiamine (vitamin $B_{1}$ ), pyridoxine (vitamin $B_{6}$ ), cyanocobalamin (vitamin $B_{12}$ ). After 9 months of therapy the patient regained muscle strength in all four limbs, without any sensory abnormalities.

\section{Conclusions}

In conclusion presented case of coexistence GuillainBarré syndrome with Landry's acute flaccid (ascending) paralysis and transverse myelitis in patient with previous history of TTP and acute post vaccination reaction may prove that the autoimmune process associated with small vessel damage and changes in the central nervous system may be a predisposition to other vascular lesions and vulnerability of CNS.

The authors declare no conflict of interest.

\section{References}

1. Bouw MC, Dors N, van Ommen H, Ramakers-van Woerden NL. Thrombotic thrombocytopenic purpura in childhood. Pediatr Blood Cancer 2009; 53: 537-542.

2. Wun T. Thrombotic thrombocytopenic purpura (TTP). Medscape, https://emedicine.medscape.com/article/206598-overview (updated: Feb 18, 2019).

3. Sauna ZE, Okunji C, Hunt RC, et al. Characterization of conformation-sensitive antibodies to ADAMTS13, the von Willebrand cleavage protease. PLoS One 2009; 4: e6506.

4. Hochberg MC, Silman AJ, Smolen JS, et al. Polyarteritis nodosa and microscopic polyangiitis. In: Rheumatology, $5^{\text {th }}$ ed. Elsevier, Philadelphia 2011; 1523-1535.

5. Hochberg MC, Silman AJ, Smolen JS, et al. Clinical features of systemic lupus erythematodes. In: Rheumatology, $5^{\text {th }}$ ed. Elsevier, Philadelphia 2011; 1229-1247.

6. Lukjanowicz M, Brzosko M. Myelitis in the course of systemic lupus erythematosus. Pol Arch Med Wewn 2009; 119: 67-73.

7. Kovasc B, Laffert T, Brent LH, et al. Transverse myelopathy in systemic lupus erythematosus; an analysis of 14 cases and review of the literature. Ann Rheum Dis 2000; 59: 120-124.

8. Birnbaum J, Petri M, Thompson R, et al. Distinct sybtypes of myelitis in sytemic lupus erythematosus. Arthritis Rheum 2009; 60: 3378-3387. 